\title{
Flexible Morphogenesis based Formation Control for Multi-Robot Systems
}

\author{
Jan Carlo Barca ${ }^{1}$, Eugene E. Lee ${ }^{2}$, Ahmet Sekercioglu \\ ${ }^{1}$ Clayton School of Information Technology, Monash University \\ ${ }^{2}$ Department of Electrical and Computer Systems Engineering, Monash University
}

\begin{tabular}{l} 
Article Info \\
\hline Article history: \\
Received Sep 17, 2012 \\
Revised Dec 2, 2012 \\
Accepted Dec 19, 2012 \\
\hline
\end{tabular}

Keyword:

Formation control Morphogenesis Multi-robot systems Networked robotics

\begin{abstract}
Inspired by how biological cells communicate with each other at a cell-tocell level; morphogenesis emerged to be an effective way for local communication between homogenous robots in multi-robot systems. In this paper, we present the first steps towards a scalable morphogenesis style formation control technique, which address the drawbacks associated with current morphogenesis type formation control techniques, including their inability to distribute robots evenly across target shapes. A series of experiments, which demonstrate that the proposed technique enables groups of non-holonomic ground moving robots to generate formations in less than 9 seconds with three robots and less than 22 seconds with five robots, is also presented. These experiments furthermore reveal that the proposed technique enables groups of robots to generate formations without significantly increasing the total travel distance when faced with obstacles. This work is an important contribution to multi-robot control theory as history has shown that the success of groups often depends on efficient and robust formation control.
\end{abstract}

Copyright (C) 2013 Institute of Advanced Engineering and Science. All rights reserved.

\section{Corresponding Author:}

First Author, Clayton School of Information Technology, Monash University Ground Floor, Building 63, Clayton Campus, Wellington Road, Clayton, Vic 3800, Australia. Email: jan.barca@monash.edu

\section{INTRODUCTION}

The study of Multi-Robot (MR) systems has recently increased in popularity due to these systems ability to i) exploit the sensing capability of large groups, ii) support high robustness toward mission failures and iii) distribute workloads amongst their members [1]. These abilities are highly sought after in a range of application areas such as search and rescue [2, 3], exploration [4, 5], surveillance [6] and environmental monitoring [7, 8]. MR systems research can therefore have a significant social and economical and impact.

Formation control is important for MR systems as history has shown that the success of groups frequently depends on the ability to generate and preserve particular formation shapes [9]. For example, circular formations enable groups to setup tight perimeters around areas of interest so that anything entering or leaving the area can be monitored, controlled or warded off. Another example is linear formations, which enables groups to move through narrow passages while staying connected or efficiently sweep across regions of interest.

Current formation control techniques for MR systems are commonly based on graph theory [10-12], potential fields [13-15] or templates [9]. Some techniques are also inspired by biological processes observed in natural systems [16]. Inspiration is often sought from nature as investigations into natural systems have proven to support development of novel rule sets, which makes it possible to solve hard problems that it would be difficult to tackle with traditional methods [1]. Additional benefits associated with seeking inspiration from natural systems include that one can investigate, test and update new theories by comparing 
them directly to the source of inspiration $[1,17]$. We therefore propose a formation control technique that is inspired by the self-organizing processes, which are guided by the molecular and cellular interactions observed in biological morphogenesis. Biological morphogenesis is discussed in greater length in [18-20].

We sought inspiration from biological morphogenesis as this process has shown an astonishing level of robustness in the natural world [21] and a great potential when applied to self-organizing swarm robotics systems [20]. However, a series of drawbacks with current morphogenesis type formation control methods also exist, including that: i) a range of these techniques only are tested in simulation [16, 22], ii) some of these techniques do not explicitly consider obstacles [23], iii) some of these techniques do not scale well with increasing numbers of robots [24], and iv) a number of these techniques are unable to guarantee that the robots are distributed evenly across their target shapes [23, 25, 26]. In this paper we present a first step towards a flexible morphogenesis type formation control mechanism that addresses these deficiencies.

The remaining parts of this paper are organized as follows. The details of the proposed formation control technique are outlined in Section 2. A series of experiments designed to quantify the performance of the technique and results from these experiments are presented in Section 3. Final conclusions and future directions of research are then presented in Section 4.

\section{RESEARCH METHOD}

The formations that ought to be generated by the robots are defined by a series of two dimensional points, which determines the shape of the formation and the centre of the formation shape. The formations can resemble simple lines, circles, triangles or square patterns, but can also have more complex $E, S$ or $C$ shapes. Points that represent linear and circular formations are illustrated in Figure 1.

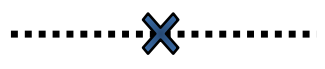

(a)

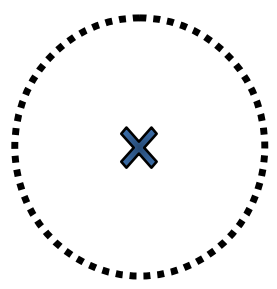

(b)

Figure 1. Two dimensional points that describe the shape of: (a) linear and (b) circular formations.

When the MR system is initialized each robot is given a distinct label that makes it possible to distinguish it from other robots. This is done by first transmitting a control signal from a base station to each individual robot. This signal makes each robot rotate for a predefined time so that the pattern on an Augmenter Reality (AR) marker mounted on top of the robot can be linked to the XBee address, which the control signal was sent to, by means of an overhead camera connected to the base station. The AR marker and the camera system are described in greater detail in Section 3.1. When the robots have been initialized they can be added or removed from the MR system without greatly affecting the system performance, as the generated formations will adapt to the number of robots observed in the system automatically at any time instant.

A human controller located at the base station determines what formation shape that will be generated by selecting a formation template, which contains the points, that describes the shape of the respective formation. A series of two dimensional Goal Points (GP) that embody the target positions for the robots are then generated on the basis of the number of points in the formation template and the current number of robots in the system.

Some formations already contain a number of predefined GP that must be covered to generate the formation shape (e.g. lines have one predefined GP in each end). If there are more robots than prespecified GP in the system, then additional intermediate GP are generated from the points that describe the target shape. In the case of a line this is done by employing Equation 1 and repeating the process with $I=2$ to $I=N_{r}-1$.

where:

$$
G_{i}=\frac{\left(I-P_{g}\right) \times N_{p}}{N_{r}-1}
$$

\footnotetext{
$G_{i} \quad$ : point on target shape to be converted to a GP

I : intermediate GP to be found

$P_{g} \quad$ : total number of required GP
} 


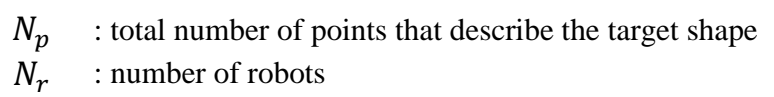

Other formations, such as circular ones, do not have any predefined GP and all the GP must therefore be generated with Equation 2. In this case the process is repeated with $I=1$ to $I=N_{r}-1$.

where:

$$
G_{i}=\frac{I \times N_{p}}{N_{r}-1}
$$

$$
\begin{array}{cl}
G_{i} & : \text { point on target shape to be converted to a GP } \\
I & : \text { intermediate GP to be found } \\
N_{p} & \text { : total number of points that describe the target shape } \\
N_{r} & \text { : number of robots }
\end{array}
$$

Each robot is assigned to its closest GP once the relevant number of GP has been generated. An overview of how this is done is provided in Figure 2.

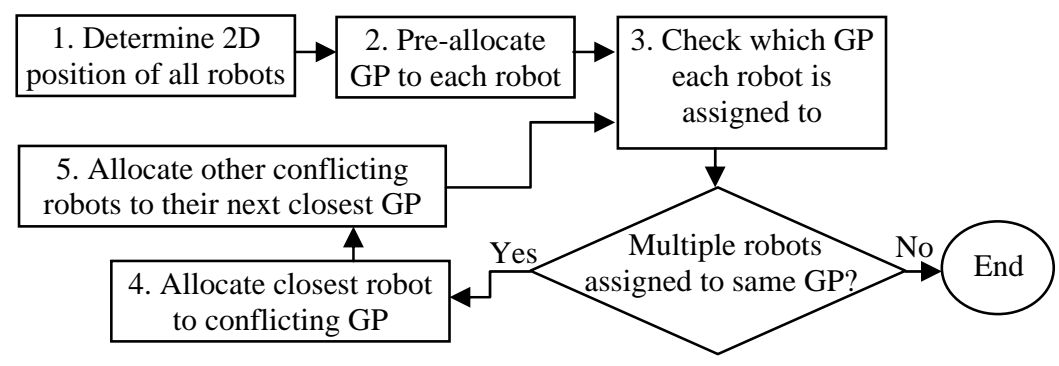

Figure 2. Overview of the GP allocation process.

In the first step of the GP allocation process each robot is assigned to its closest GP by means of the Sum Squared Distance (SSD) measure (Equation 1). The system then checks if multiple robots were assigned to the same GP. If this is the case, then the relevant robots compete to determine who is closest to the GP at hand. Robot/s that looses this competition is assigned to their next closest GP. This process is repeated iteratively until no conflicting GP is present and ensures that the total travelling distance of the robots can be kept at a minimum.

where:

$$
D=a b s\left(X_{c}-X_{d}\right)^{2}+a b s\left(Y_{c}-Y_{d}\right)^{2}
$$

$$
\begin{array}{cl}
D & : \text { SSD } \\
\left(X_{c}, Y_{c}\right) & : \text { current position } \\
\left(X_{d}, Y_{d}\right) & : \text { desired position }
\end{array}
$$

Once a unique GP is assigned to each robot the robots either: i) rotate towards their assigned GP, ii) move towards their GP, or iii) avoid obstacles. Rotation is triggered when the angle between the heading direction of the robot and the position of the GP is greater than $R_{n}$ or smaller than $R_{p}$. The shortest direction of rotation is selected as in [9] to ensure that the associated energy consumption can be minimized. We also employ the Inverse Square Distance Law (ISDL) shown in Equation 4 to produce a resistive force that reduces the rotational velocity of the robot as it approaches the desired angle, and an error count value $C_{e}$, which forces the robot to stop rotating when it has oscillated more than $C_{e}$ times, to inhibit oscillation.

where:

$$
R \propto \frac{1}{D^{2}}
$$

$$
R \quad \text { : resistive force }
$$


When the robot has acquired its desired trajectory it advances towards its GP, and once again the robot is governed by a velocity control function that makes use of the ISDL. However, this time the velocity is inversely proportional to the SSD to either another robot or to the GP, depending on which of them that is closest to the robot at hand. This is done to prevent the robots from overshooting their GP and to reduce the number of collisions.

Collision avoidance is triggered when another robot enters a quadrant on a sensing disk, which is centered on the robot at hand, and the GP of the robot at hand is located in a neighbouring quadrant. The employed quadrant system is illustrated in Figure 3, and a situation that triggers collision avoidance is illustrated in Figure 4.

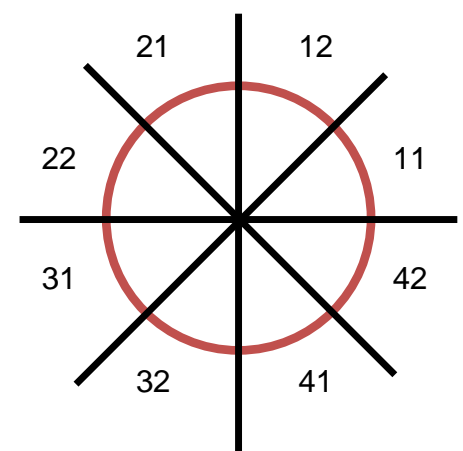

Figure 3. Quadrant system.

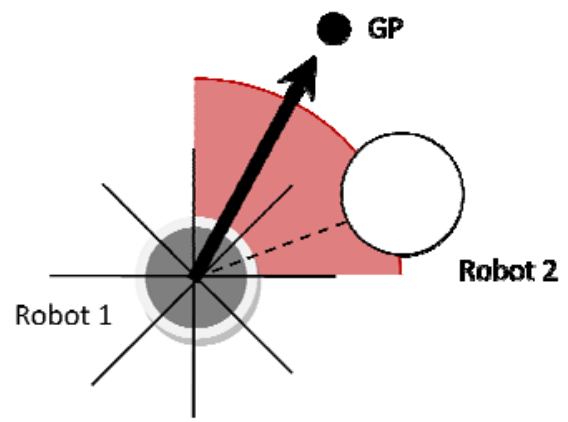

Figure 4. A situation that triggers collision avoidance.

The robots that are about to collide compare how far from their respective GP they are located when collision avoidance is triggered. The robot that is closest to its associated GP will then cease to move, while the other robot generates a new Temporary Goal Point (TGP). This TGP is generated $T_{d}$ away from the robot at hand in a direction that reflects the average angle in the closest quadrant with no colliding nodes. The robot will then rotate in the manner as described above and advance toward its TGP. The robot continues to move toward its original GP when the TGP is reached.

When a robot reaches it's GP it remains still until: i) the number of robots in the MR system changes, or ii) the base station commands the robots to generate a new formation shape.

\section{RESULTS AND ANALYSIS}

Experiments that quantify the performance of the proposed formation control technique on eBug robots are presented in this section. The experiments are carried out on linear and circular formations due to their wide applicability. E.g. potential application areas for linear formations include border monitoring and efficient sweep through areas of interest, while circular formations can be used to protect moving convoys, surround enemy targets or to set up perimeters around sites such as atom reactors and chemical plants in order to efficiently detect pollution [9].

The performance criterions used in the experiments are: i) convergence time, ii) travel distance and iii) number of collisions. Convergence time is used as a criterion because relevant formations must be generated quickly in order to efficiently address tasks at hand, while travel distance is included as a criterion 
because movement greatly contributes to energy consumption and robots that run out of battery are useless. The number of collisions is used as a criterion because collisions potentially can damage the robots.

The presented experiments were carried out by first separating a Region of Interest (RoI) into an 8x8 grid and generating random initial starting positions for three robots located within the grid system. The performance of the formation control technique was then measured ten times with different random starting positions assigned to each robot, before the average results were calculated. The experiments were also repeated with four and five robots to investigate if the MR system is scalable. The maximum number of robots used in the experiments was set to five due to space limitations. Implementation specific parameters are presented in Table 1.

Table 1. Implementation specific parameters.

\begin{tabular}{cc}
\hline Variable & Value \\
\hline $\mathrm{R}_{\mathrm{n}}$ & $-5^{\circ}$ \\
$\mathrm{R}_{\mathrm{p}}$ & $5^{\circ}$ \\
$C_{e}$ & 5 \\
$T_{d}$ & $18.75 \mathrm{~cm}$ \\
\hline
\end{tabular}

The hardware that was used in the experiments is briefly described in Section 3.1 before we go on to describe the results from the experiments in Section 3.2.

\subsection{Hardware}

The eBug robot (Figure 5), which originally was designed by Nick D’Ademo, is used to quantify the performance of the proposed formation control technique. The key components of the eBug include: i) an XBee chip, which provides the robot with a unique XBee address, and enables the robot to wirelessly communicate ZigBee packets to other eBugs and a base station, ii) a Printed Circuit Board (PCB) and a LiPo battery that supplies power, iii) a chassis made up of two stepper motors and two wheels, iv) a logic PCB responsible for data processing, and v) an $11 \mathrm{~cm}$ wide AR marker, which is placed on the top of the robot, and provides the eBug with a unique identification label that can be observed by an overhead Playstaion Eye camera that tracks the two dimensional pose of the robot within the RoI over time.

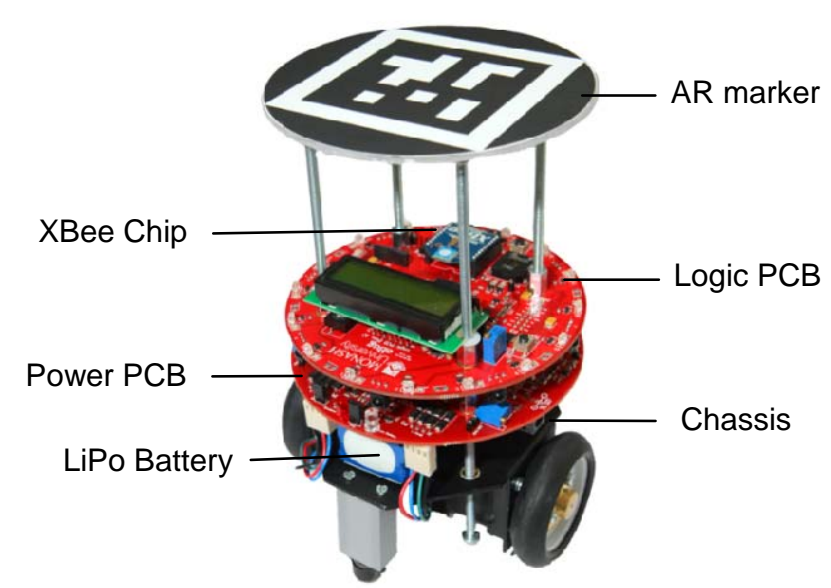

Figure 5. The eBug robot.

\subsection{Convergence Time}

Results from the experiment that measures the convergence time of the eBugs as linear and circular formations are generated, are discussed in this section. An overview of how the average convergence time increases as the number of robots are raised from three to five is provided in Figure 6. 


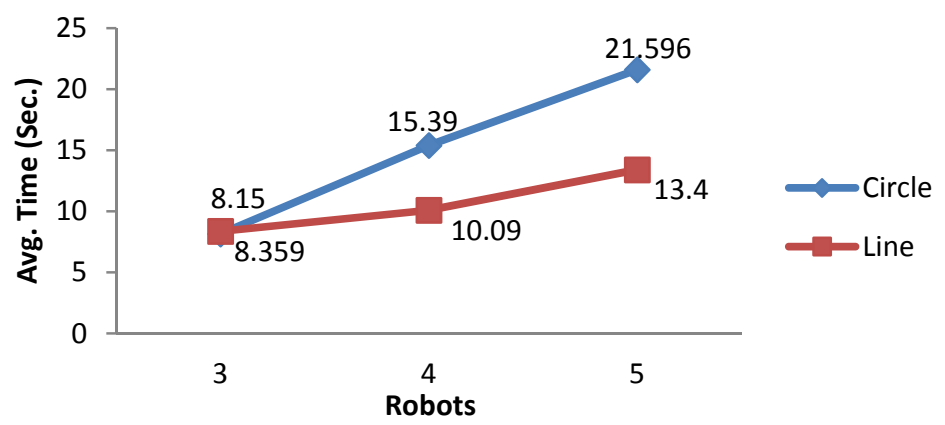

Figure 6. Convergence time when linear and circular formations are generated.

One can observe that both linear and circular formations are generated in less than 9 seconds with three robots and less than 22 seconds with five robots. By studying the graphs one can also observe that the convergence time increases with $60 \%$ when linear formations are generated and with 165\% when circular formations are generated and the number of robots is raised. This shows that one can expect to produce linear formations faster than circular when the proposed formation control technique is employed, which is an important insight in situations where formations must be generated fast in order to address issues at hand.

\subsection{Travel Distance}

Outcomes from the experiments that meaure the travel distance of the eBug robots as they generate linear and circular formations with the proposed formation control technique are described in this section. Graphs that represent the average travel distance of the eBugs as the number of robots is increased are shown in Figure 7.

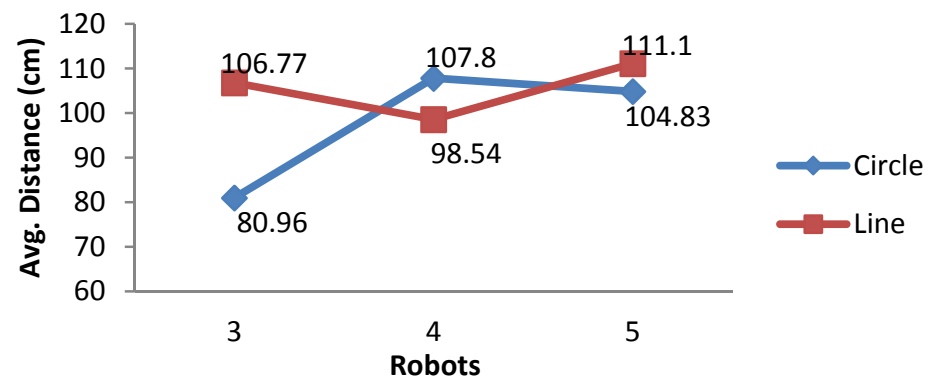

Figure 7. Travel distance when linear and circular formations are generated.

By analyzing the graphs one find that the average travel distance only increases with $4 \%$ when the number of robots is raised from three to five in linear formations, and that the distance increases with $29 \%$ when the number of robots is raised in circular formations. One also find that the total average travel distance is 108.55 $\mathrm{cm}$ for the linear formations and $97.86 \mathrm{~cm}$ for the circular formations. This shows that it is more energy efficient to generate circular than linear formations. One should therefore consider generating the former in situations where battery power is limited and a particular issue can be addressed by both formation shapes.

\subsection{Collisions}

Results from the experiments that gauge the number of collisions, which occur between the eBug robots as circular and linear formations are generated, are described in this section. In Figure 8 one can observe how the number of collisions increase as the number of robots is raised from three to five. 


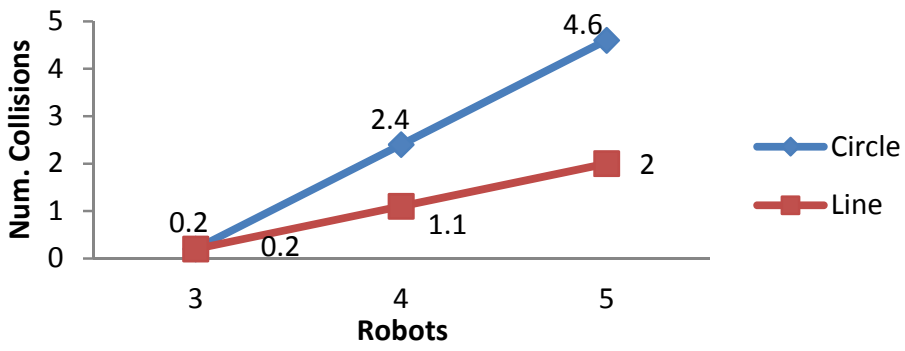

Figure 8. Number of collisions when linear and circular formations are generated.

The graphs show that there are few collisions when there are three robots in the system, and that the number of collisions increases linearly as the number of robots is raised to five. This is expected as the RoI becomes increasingly congested when the number of robots is raised. However, it is important to notice that this does not inhibit the robots from generating the formations, and it does not severely affect their travel distance. The latter is evident as the robots managed to generate circular formations with a smaller average travel distance than linear formations, even if the number of collisions were higest when circular formations were produced.

\subsection{Additional Preliminary Experiments}

It is important to note that the formation control technique not is limited to the formations used in the above experiments. Preliminary trials have shown that S, E and C shaped formations also can be produced (Figure 9). A video that shows how these formations are generated can be observed at: http://wsrnlab.ecse.monash.edu.au/publications.html.

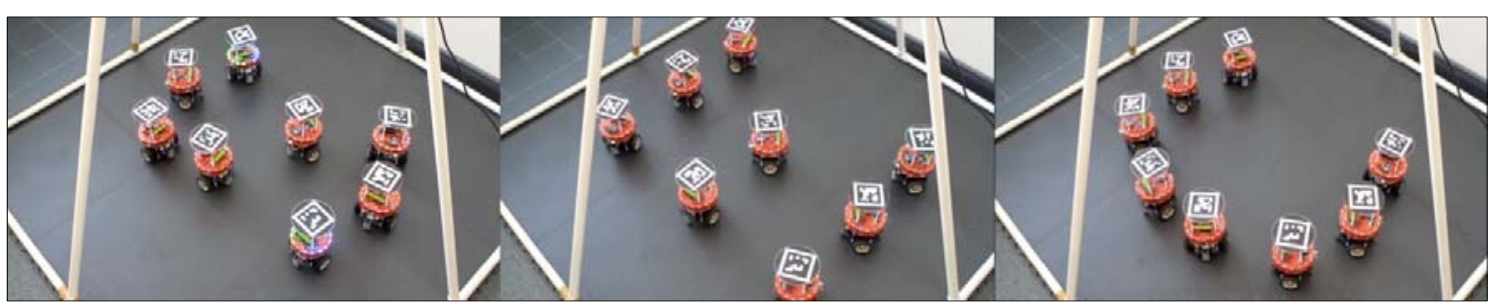

Figure 9. $S, E$, and $C$ shaped formations that have been generated in preliminary trials.

\section{CONCLUSION}

The first step towards a scalable and robust morphogenesis inspired formation control technique that address the drawbacks with current morphogenesis type formation control techniques have been presented. Results from a series of experiments carried out on eBugs demonstrate that the proposed technique enables groups of non-holonomic ground moving robots to generate circular and linear formations in less than 9 seconds with three robots, and less than 22 seconds with five robots. The experiments also show that the proposed technique enables robots to generate formations without greatly affecting the travel distance when faced with obstacles. The limitations of this research are: i) that a central base station and global data was used to facilitate processing, and ii) that the current size of the RoI prevented us from carrying out experiments on large numbers of robots. Future research will address these limitations.

\section{ACKNOWLEDGEMENTS}

This research was supported in part by Lise and Arnfinn Hejes Grant for Education and Research. We would like to thank Adv. Harald Røer for the way he administered the grant.

\section{REFERENCES}

[1] J. C. Barca and A. Sekercioglu, "Swarm Robotics Reviewed," Robotica, Available on CJO 2012 doi:10.1017/S026357471200032X, pp. 1-32, 2012. 
[2] S. Martinez, et al., "Motion Coordination with Distributed Information,” IEEE Control Systems Magazine, vol. 27, pp. 75-88, 2007.

[3] J. Jung, et al., "Multi-Robot Path Finding with Wireless Multihop Communications," IEEE Communications Magazine, pp. 126-132, 2010.

[4] G. Hinchey, et al., "Swarms and Swarm Intelligence," Computer, vol. 40, pp. 111-113, 2007.

[5] M. Ma and Y. Yang, “Adaptive Triangular Deployment Algorithm for Unattended Mobile Sensor Networks,” IEEE Transactions on Computers, vol. 56, pp. 946-958, 2007.

[6] P. Vincent, et al., "Analysis for Cooperative Search Using UAV Swarms,” in ACM Symposium on Applied Computing, Nicosia, pp. 79-86, 2004.

[7] X. Li, et al., "Localized Self-Deployment of Mobile Sensors for Optimal Focused-Coverage Formation,” Technical Report, Carleton University, pp. 1-18, 2008.

[8] J. Cortés and F. Bullo, "Coordination and Geometric Optimization via Distributed Dynamical Systems," Journal on Control and Optimization, vol. 44, pp. 1543-1574, 2005.

[9] J. C. Barca and A. Sekercioglu, "Generating Formations with a Template based Multi-Robot System,” Australasian Conference on Robotics and Automation (ACRA), Melbourne, pp 1-9, 2011.

[10] J. C. Barca, et al., "Controlling Formations of Robots with Graph Theory,” 12th International Conference on Intelligent Autonomous Systems (IAS), Jeju Island, pp 1-8, 2012.

[11] J. Desai, et al., "Modeling and Control of Formations of Nonholonomic Mobile Robots,” Trans. on Robotics and Automation, vol.17, pp. 905-908, 2001.

[12] B. Fidan, et al., “Acquiring and maintaining persistence of autonomous multi-vehicle formations,” IET Control Theory \& Applications, vol. 1, pp. 452-460, 2007.

[13] V. Gazi and K. Passino, Swarm Stabillity and Optimization, Springer, doi:10.10007/978-3-642-18041-5, 2011.

[14] Z. Xue and J. Zeng, "Formation Control Numerical Simulations of Geometric Patterns for Unmanned Autonomous Vehicles with Swarm Dynamical Methodologies," International Conference on Measuring Technology and Mechatronics Automation, Zhangjiajie, pp. 477 - 482, 2009.

[15] L. Barnes, et al., "Unmanned ground vehicle swarm formation control using potential fields," Mediterranean Conference on Control \& Automation, Athens, pp. 1-8, 2007.

[16] M. Mamei, et al.,"Experiments of Morphogenesis in Swarms of Simple Mobile Robots,” Applied Artificial Intelligence, vol. 18, pp. 903-919, 2004.

[17] B. Adams, et al., "Humanoid robots: a new kind of tool," IEEE Intelligent Systems \& control letters, vol. 15, pp. 25-31, 2004

[18] L. Beloussov, "Integrating self-organization theory into an advanced course on morphogenesis at Moscow State University,” International Journal on Developmental Biolology, vol. 47, pp. 177-181, 2003.

[19] K. Simons, et al.,"Self-Organization and Morphogenesis in Biological Systems”, Self-organization in Biological Systems, S. Camazine et al., (Eds.), Schloss Ringberg, Tegersee, 2006.

[20] Y. Jin, and Y. Meng, “Morphogenetic Robotics: An Emerging New Field in Developmental Robotics,” IEEE Trans. on Systems, Man and Cybernetics, Part C: Applications and Reviews, vol 46, pp. 145-160, 2010.

[21] H. Ben-Amor, et al.,"Regulatory networks analysis: Robustness in morphogenesis," Proc. of International Conference on Advanced Information Networking Applications Workshops, Bradford, pp. 924-928, 2009.

[22] H. Sayama, "Robust Morphogenesis of Robotic Swarms," IEEE Computational Intelligence Magazine, vol. 5, pp. 43-49, 2010.

[23] H. Guo, "Analysis of local communication load in shape formation of a distributed morphogenetic swarm robotic system,” IEEE Congress on Evolutionary Computation (CEC), Barcelona, pp. 1-8, 2010.

[24] H. Guo, et al.,"Swarm Robot Pattern Formation using a Morphogenetic Multi-Cellular based Self-organizing Algorithm," IEEE International Conference on Robotics and Automation, pp. 3205-3210, 2011.

[25] L. Chaimowicz, et al.," Controlling Swarms of Robots Using Interpolated Implicit Functions," Proceedings of the 2005 IEEE International Conference on Robotics and Automation, pp. 2487 - 2492, 2005.

[26] Y. Jin, , et al., "A morphogenetic self-organization algorithm for swarm robotic systems using relative position information,” UK Workshop on Computational Intelligence (UKCI), Colchester pp. 1-6, 2010.

\section{BIOGRAPHIES OF AUTHORS}

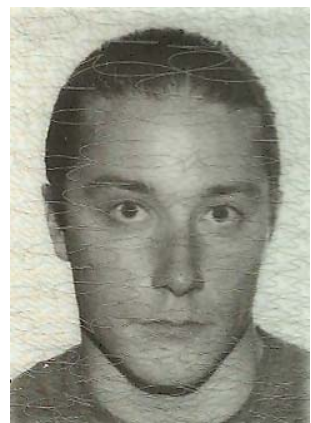

Dr. Jan Carlo Barca received his PhD in Information Technology from Monash University, Australia, in 2009. He is currently a co-director of the Wireless Sensors and Robot Networks (WSRN) Laboratory, a Research Fellow and a member of the Centre for Research in Intelligent Systems (CRIS) at Monash University. His major research interests are in the areas of swarm robotics, control of multiple quadcopters and intelligent ground vehicles, computer vision, computational intelligence and interactive 3D simulations. Jan Carlo has published work at several international conferences and in high level journals. He has also managed projects in industry and academia, including a project with a research budget of $\$ 259000,-$. Throughout his career he has received several grants including the Norwegian King’s Council (Hoffjegermester Sund and Kabinetskammerherre Ferd. Egebergs) study grant. His e-mail address is jan.barca@monash.edu and his website can be found at http://www.jancarlobarca.com 


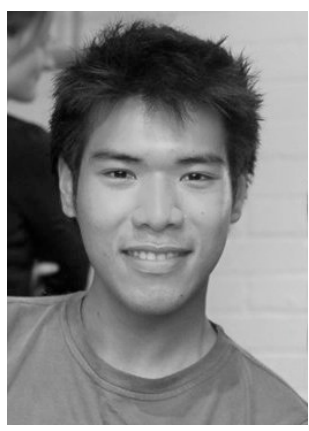

Mr. Eugene Eu-Juin Lee graduated as an Electrical and Computer Systems Engineer from Monash University, Australia, in 2012. Having completed his Cambridge IGCSE and Cambridge A Levels qualifications at the Britisih International School Shanghai, he started his engineering undergraduate study at Monash University, Sunway campus in Malaysia, prior to continuing his degree at Monash University, Clayton campus in Australia. Whilst at university, Eugene was awarded the recognition on the Dean's Honours list, Merital Scholarship by the university, and was a member of the Monash Golden Key Society. He was also awarded with the Braemec Altera Award for Best Group Project, for having built the most innovative stairs climbing robot. Having completed his engineering degree, Eugene is now an Electrical Engineer working in the mining industry with Rio Tinto. His e-mail address is lee.eujuin@gmail.com and his professional career profile can be found at http://www.linkedin.com/in/eujuinlee.

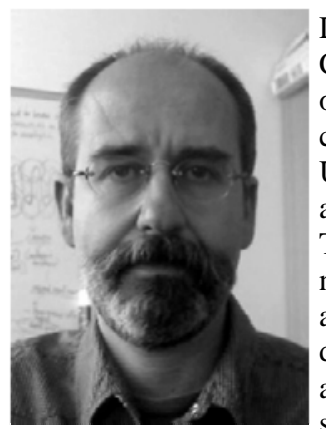

Dr. Ahmet Sekercioglu is a member of the academic staff at the Department of Electrical and Computer Systems Engineering of Monash University, Melbourne, Australia. He was the leader of the Applications Program of Australian Telecommunications CRC until the completion of the centre's research activities (December 2007). He has completed his PhD degree at Swinburne University of Technology, BSc and MSc degrees (all in Electrical and Electronics Engineering) at Middle East Technical University, Ankara, Turkey. He lectured at Swinburne University of Technology, Melbourne, Australia for eight years. Prior to his academic career, he held numerous positions as a research engineer in private industry. He has published 14 journal articles, 2 book chapters, 56 conference papers and has filed 2 patents. His recent research is in distributed algorithms for self-organization in wireless networks. He is also working in the application of intelligent control techniques for multiservice networks as complex, distributed systems. His e-mail address is ASekerci@ieee.org and his Web site can be found at http://titania.ctie.monash.edu.au. 